\title{
An Elderly Man with Mediastinal Mass and Sepsis
}

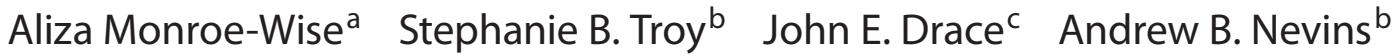 \\ a Stanford University School of Medicine and bivision of Infectious Diseases and Geographic Medicine, \\ Stanford University School of Medicine, Stanford, Calif., and ${ }^{\mathrm{C}}$ Radiology Service, Veterans Affairs Palo Alto Health \\ Care System, Palo Alto, Calif., USA
}

\section{Case Report}

An 81-year-old man with a history of colon cancer presented with progressive hoarse voice of 6 months' duration. He denied dysphagia, odynophagia, cough, weight loss, fevers, or chills. A flexible laryngoscopy revealed left-sided vocal chord paralysis. Computed tomography (CT) scan with contrast showed a $4 \times 4 \times 3 \mathrm{~cm}$ mediastinal mass located directly below the aortic arch and aortic root dilation measuring $5 \mathrm{~cm}$ (fig. 1). He underwent bronchoscopy for tissue diagnosis; during this procedure, the endobronchial ultrasound (EBUS) failed, and so a blind transtracheal biopsy was performed, for which pathology was nondiagnostic. One week later, he underwent a second bronchoscopy with multiple EBUS-guided transtracheal biopsies of the mass. Pathologic examination was again nondiagnostic, revealing a small fragment of cartilage, hypocellularity, and no evidence of neoplasm. Gram stain taken during the second bronchoscopy showed no white blood cells (WBC) or organisms, but cultures subsequently grew rare Streptococcus salivarius and Streptococcus mitis.

Four days after the second bronchoscopy, a positron emission tomography (PET)/CT scan was performed (fig. 2) that showed significant metabolic activity in a rim surrounding the mass, the center of which was metabolically inactive. New mediastinal edema and enlarged, hypermetabolic lymph nodes were also noted. As a result of this scan, the patient was directly admitted for further evaluation. At that time he was asymptomatic except for the hoarse voice and chronic back pain.

On admission the patient was afebrile with no significant findings on physical examination. His WBC count
Fig. 1. Initial CT.

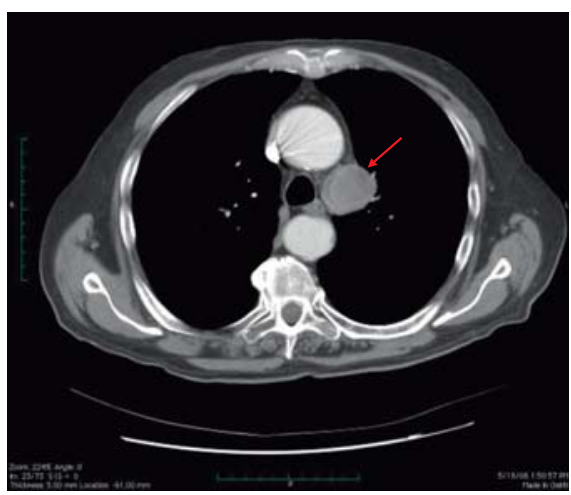

Fig. 2. PET/CT scan.

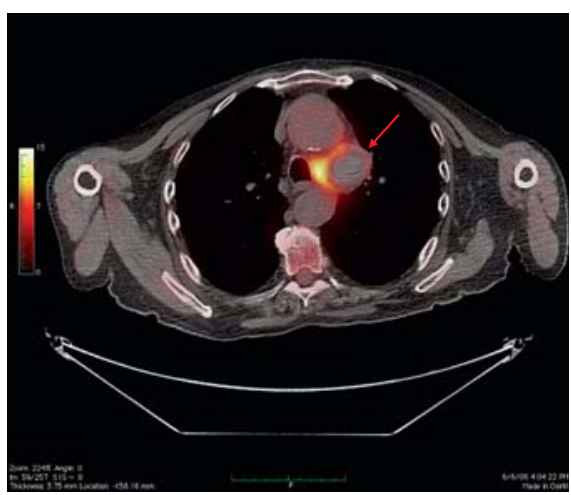

was $12.9 \times 10^{9} / 1$. The morning following admission, however, his systolic blood pressure dropped to the 70s, he became tachycardic, and his WBC count rose to $17 \times$ $10^{9} / 1$. He was transferred to the ICU and given vasopressors and broad-spectrum antibiotics. A CT angiogram (CTA) showed no evidence of aortic dissection or significant vascular connection to the mass. Blood cultures grew Gram-positive cocci in pairs, chains, and groups.

What is your diagnosis?

\section{KARGER}

Fax +4161306 1234

E-Mail karger@karger.ch

www.karger.com (c) 2010 S. Karger AG, Basel

0025-7931/10/0802-0157\$26.00/0

Accessible online at:

www.karger.com/res
Dr. Stephanie Troy

Division of Infectious Diseases and Geographic Medicine

Stanford University Medical Center, 300 Pasteur Drive

Grant Building S101 (MC 5107), Stanford, CA 94305-5107 (USA)

Tel. +1 650725 8338, Fax +1 650498 7011, E-Mail sbtroy@stanford.edu 


\section{Diagnosis: Three Aspects Are Discussed}

\section{Radiographic Diagnosis}

\section{Superinfected Thrombosed Ductus Arteriosus Aneurysm}

The diagnosis of thrombosed ductus arteriosus aneurysm was made based on 3-dimensional imaging that showed a close association between the mass and both the aortic arch and the pulmonary artery (fig. 3). The mass was situated in the precise anatomic location of the ligamentum arteriosum or a patent ductus arteriosus. The lack of contrast in the mass during the CTA suggested that the aneurysm was thrombosed. In addition, the mass was radiographically hyperdense, which is consistent with blood or tissue but not with a cyst, and the mass also had a homogeneous appearance not consistent with a teratoma. In speaking with the pulmonologist who performed the biopsies, the aspirate seemed solid, and there was no evidence of pus. In reviewing the pathology slides, there was no evidence of a primary infectious process.

Traditionally, mediastinal masses are classified by their anatomic location in the anterosuperior, middle, or posterior compartment. The most common masses found in the anterosuperior compartment are thymomas, lymphomas, teratomas, and thyroid tumors [1]. Aortic aneurysms can arise in any compartment of the mediastinum.

A 1991 review described 144 cases of ductus arteriosus aneurysms reported in the literature, of which 32 (30\%) occurred in adults [2]. 74\% of cases occurred spontaneously, the remainder being associated with surgery. It has been postulated that the etiology behind the majority of spontaneous ductus arteriosus aneurysms in adults may be delayed closure of the aortic end of the ductus, resulting in an aortic diverticulum which may enlarge over time with exposure to systemic pressure [2]. Hoarseness due to compression of the left recurrent laryngeal nerve is a common presenting symptom, as are cough, anorexia, and pain [3-6]. Diagnosis is primarily radiographic, with three-dimensional imaging, including CT and MRI, being particularly useful [7]. Potential complications that have been reported include spontaneous rupture, erosion, superinfection, and thrombosis [2, 8]. Management is surgical, and can require cardiopulmonary bypass [2], although endovascular repair has also been described [9].

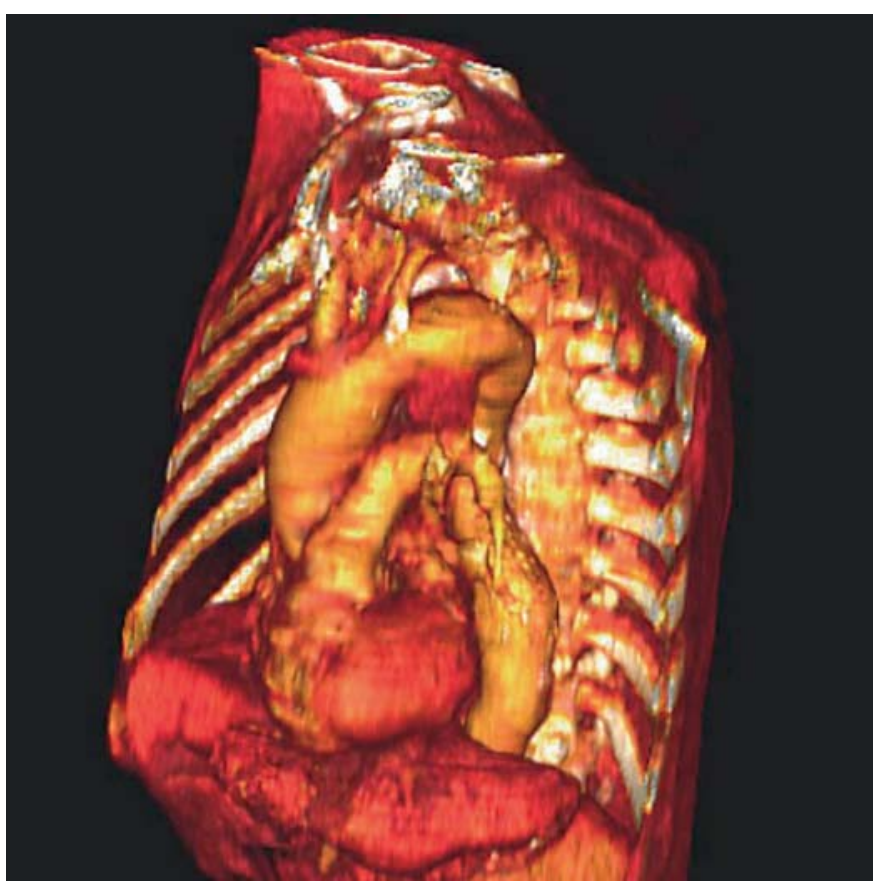

Fig. 3. Three-dimensional image.

\section{Pathological Diagnosis}

\section{Superinfected Thrombosed Pulmonary Vascular Malformation}

Our patient died 4 months later due to unrelated causes. Findings on autopsy were not consistent with the clinical and radiographic diagnosis, as the mass was connected to neither the aorta nor the pulmonary artery. Instead, it was part of the left upper lobe of the lung, although it was wedged between the aorta and the pulmonary artery in the space usually occupied by the ligamentum arteriosum. Histologic examination of the mass tissue revealed a large encapsulated fibrin clot with evidence of scarring and inflammation. There was no evidence of granulomatous inflammation, and Congo red stain did not detect the presence of amyloid. Gomori methanamine silver, acid-fast bacilli, Gram, and periodic acid-Schiff with diastases digestion stains were also negative, excluding the presence of microorganisms. However, the combination of trichrome stains and a Verhoeffvan Gieson stain positive for elastic tissue suggested a disrupted vascular wall (fig. 4). These findings were consistent with a thrombosed vascular malformation. 
Vascular malformations include arteriovenous, venous, and capillary malformations, hemangiomas, angiolipomas, and combined vascular malformations. Vascular malformations may be congenital, acquired, or induced by trauma [10]. Treatment commonly involves embolization [11], and surgical excision may also be required [12]. In this patient, histology from the autopsy was insufficient to determine the type of vascular malformation. Although the Verhoeff-van Gieson and trichrome stains clearly indicated that the mass was vascular in nature, the lack of clear connection to a vein precluded a definitive diagnosis of arteriovenous malformation. Although the vascular malformation may have been present for some time, the patient's recent development of left-sided vocal chord paralysis was most likely due to shifts in the length and shape of the mediastinum that can occur with age.

\section{latrogenic Superinfection}

While the nature of the mass was not discovered until autopsy, clinical and radiographic evidence supported superinfection as the etiology of this patient's sepsis. Mediastinal infections most commonly result from contiguous extension from the oropharynx, esophageal rupture, or complications from cardiothoracic surgery. More rarely, Histoplasma capsulatum and other fungi, Mycobacterium tuberculosis, and Bacillus anthracis can cause infections in the mediastinum [13]. Our patient's clinical picture was not consistent with these infections, and his mass preceded any evidence of infection.

Comparisons between the initial CT scan (fig. 1) and the subsequent PET/CT scan (fig. 2) and CTA performed 2 weeks later provided the diagnosis of superinfection of the mediastinal mass. The initial CT scan showed no enlarged lymph nodes or inflammatory stranding, but the PET/CT scan and the CTA showed significant lymphadenopathy, mediastinal edema, and inflammatory stranding. The blood cultures drawn when the patient was septic ultimately grew the same $S$. salivarius and $S$. mitis cultured from the second biopsy as well as nutritionally-deficient Streptococcus. Given the timeline, the radiographic evidence, and the nature of these organisms as components of the normal oral flora, it is likely that the infection was introduced iatrogenically as a result of multiple transbronchial biopsies. Although no complications of EBUS fine-needle aspiration have been reported in the literature $[14,15]$, infection is a rarely reported complication of transbronchial biopsy $[16,17]$. There have been no
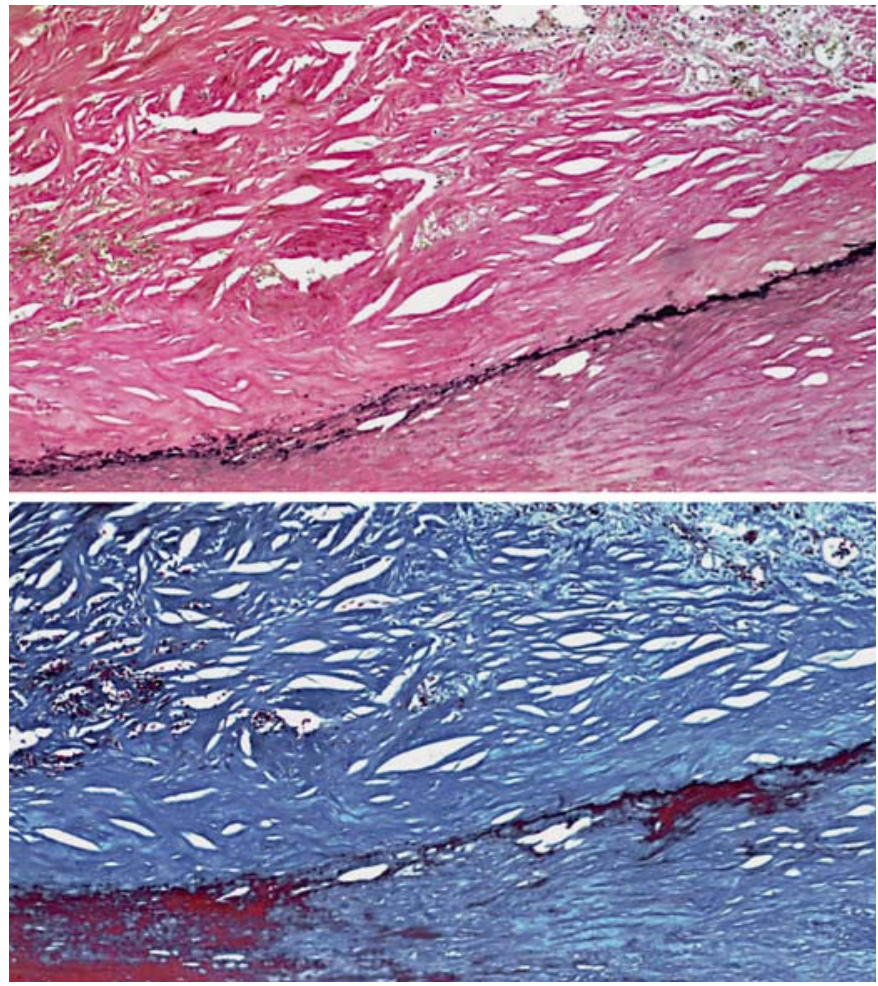

Fig. 4. Histologic images of the mass.

previously reported cases of sepsis resulting from biopsyintroduced infection of a vascular malformation located in the mediastinum.

Given our patient's age and comorbidities, it was determined that he was not a candidate for surgical intervention. His infection was treated with a prolonged course of intravenous antibiotics. At follow-up 3 months after recovering from his initial septic episode, he remained asymptomatic except for his persistent hoarse voice. Unfortunately, 1 month later, he died from a small bowel obstruction. At autopsy, all signs of infection had resolved.

\section{Acknowledgment}

We are indebted to Dr. Jon Kosek of the Pathology Department at the Veterans Affairs Palo Alto Health Care System for providing the histology slides and the autopsy results.

\section{Key Words}

Mediastinal mass · Sepsis · Superinfection •

Vascular malformation - Ductus arteriosus aneurysm 


\section{References}

$\checkmark 1$ Mullen B, Richardson JD: Primary anterior mediastinal tumors in children and adults. Ann Thorac Surg 1986;42:338-345.

$\checkmark 2$ Lund JT, Jensen MB, Hjelms E: Aneurysm of the ductus arteriosus. A review of the literature and the surgical implications. Eur J Cardiothorac Surg 1991;5:566-570.

-3 Hirose H, Takagi M, Kugimiya T, Miyagawa N, Hashiyada H, Noguchi M, Tada S: Spontaneously developed aneurysm of the ductus arteriosus in an adult. Ann Vasc Surg 1999, 13:229-231.

4 Pastuszko P, Eisenberg JA, Diehl JT: Ductus arteriosus aneurysm in an adult patient presenting with hoarseness. J Card Surg 2005; 20:386-388.

$\checkmark 5$ Day JR, Walesby RK: A spontaneous ductal aneurysm presenting with left recurrent laryngeal nerve palsy. Ann Thorac Surg 2001; 72:608-609.

6 Vlachou P, Karkos C, Vaidhyanath R, Entwisle J: Ortner's syndrome: an unusual cause of hoarse voice. Respiration 2008; 75 : 459-460.
7 Jan SL, Hwang B, Fu YC, Chi CS: Initial clinical experience of real-time three-dimensional echocardiography in neonates with isolated congenital ductus arteriosus aneurysm. Int J Cardiol 2005;105:300-305.

8 Dyamenahalli U, Smallhorn JF, Geva T, Fouron JC, Cairns P, Jutras L, Hughes V, Rabinovitch M, Mason CA, Hornberger LK: Isolated ductus arteriosus aneurysm in the fetus and infant: a multi-institutional experience. J Am Coll Cardiol 2000;36:262-269.

$\checkmark 9$ Saito N, Kimura T, Toma M, Teragaki M, Minaminimura H, Kita T, Inoue K: Successful endovascular repair of an aneurysm of the ductus diverticulum with a branched stent graft: case report and review of literature. J Vasc Surg 2004;40:1228-1233.

10 Gutierrez F, Monaco M, Hartmann AJ, McKnight R: Congenital arteriovenous malformations between brachiocephalic arteries and systemic veins. Chest 1987;92:897-899.

11 Post M, van Gent M, Snijder R, Mager J, Schonewille W, Plokker H, Westermann C: Pulmonary arteriovenous malformations and migraine: a new vision. Respiration 2008;76:228-233.
12 Grillo H, Athanasoulis C: Tracheal obstruction from mediastinal arteriovenous malformation. J Thorac Cardiovasc Surg 2004;128: 780-782.

13 Rupp M: Mediastinitis; in Mandell G, Bennett J, Dolin R (eds): Mandell, Douglas, and Bennett's Principles and Practice of Infectious Diseases. Philadelphia, Elsevier, 2005.

14 Yasufuku K, Nakajima T, Chiyo M, Sekine Y, Shibuya K, Fujisawa T: Endobronchial ultrasonography: current status and future directions. J Thorac Oncol 2007;2:970-979.

15 Herth F, Eberhardt R, Ernst A: The future of bronchoscopy in diagnosing, staging and treatment of lung cancer. Respiration 2006 ; 73:399-409.

16 Pereira W Jr, Kovnat DM, Snider GL: A prospective cooperative study of complications following flexible fiberoptic bronchoscopy. Chest 1978;73:813-816.

17 Ibrahim AS, Allangawi MH, Sattar HA, Mobyed HS, Almohammed AA: Indications, diagnostic yields and complications of transbronchial biopsy over 5 years in the State of Qatar. Saudi Med J 2005;26:641-645. 
